\title{
Gut-derived Lipopolysaccharide Promotes Alcoholic Hepatosteatosis and Subsequent Hepatocellular Carcinoma by Stimulating Neutrophil Extracellular Traps Through TLR4
}

Yang Liu ( $\square$ liu-yang@xjtu.edu.cn )

Xi'an Jiaotong University Second Affiliated Hospital https://orcid.org/0000-0002-5463-0791

Xin Zhang

Xi'an Jiaotong University Second Affiliated Hospital

Shuo Chen

Xi'an Jiaotong University Second Affiliated Hospital

Jiazhong Wang

Xi'an Jiaotong University Second Affiliated Hospital

Shuo Yu

Xi'an Jiaotong University

Yiming Li

Xi'an Jiaotong University Second Affiliated Hospital

Meng Xu

Xi'an Jiaotong University Second Affiliated Hospital

Harouna Aboubacar

Xi'an Jiaotong University

Junhui Li

Xi'an Jiaotong University Second Affiliated Hospital

Tao Shan

Xi'an Jiaotong University Second Affiliated Hospital

Jixin Wang

Xi'an Jiaotong University Second Affiliated Hospital

Gang Cao

Xi'an Jiaotong University Second Affiliated Hospital

\section{Research Article}

Keywords: Neutrophil extracellular traps, alcoholic, hepatosteatosis, hepatocellular carcinoma, LPS, TLR4

Posted Date: December 9th, 2021 
DOI: https://doi.org/10.21203/rs.3.rs-1124551/v1

License: (c) (1) This work is licensed under a Creative Commons Attribution 4.0 International License. Read Full License 


\section{Abstract}

\section{Background}

Binge drinking leads to many disorders, including alcoholic hepatosteatosis, which is characterized by intrahepatic neutrophil infiltration and which increases the risk of hepatocellular carcinoma (HCC). Molecular mechanisms may involve the migration of bacterial metabolites from the gut to the liver and activation of neutrophil extracellular traps (NETs).

\section{Methods}

Serum from both binge drinking and alcohol-avoiding patients was analyzed. Mouse models of chronical plus binge alcohol indued steatosis and HCC models were used.

Results

A marker of NETs formation, lipopolysaccharide, was significantly higher in alcoholic steatosis and HCC patients and mice than in controls. Intrahepatic inflammation markers and HCC-related cytokines were decreased in mice with reduced NET formation due to neutrophil elastase (NE) deletion, and liver-related symptoms of alcohol were also alleviated in NE KO mice. Removal of intestinal bacteria with antibiotics led to decreases in markers of NETs formation and inflammatory cytokines upon chronic alcohol consumption, and genesis of alcoholic hepatosteatosis and HCC was also attenuated. These functions were restored upon supplementation with the bacterial product lipopolysaccharide (LPS). When mice lacking TLR4 received chronic alcohol feeding, intrahepatic markers of NETs formation decreased, and hepatosteatosis and HCC were alleviated.

\section{Conclusion}

Formation of NETs following LPS stimulation of TLR4 upon chronic alcohol usage leads to increased alcoholic steatosis and subsequent HCC.

\section{Background}

Alcohol consumption is becoming a leading risk factor impacting population health worldwide. According to an annual report of the World Health Organization, although the global percentage of drinkers has declined, the total alcohol consumption per capita has been increasingly steadily since $2000^{1}$. In 2018 , worldwide annual average consumption was the equivalent of $6.4 \mathrm{~L}$ of pure alcohol per person; when considering only people classified as "drinkers," the average consumed increases to $15.1 \mathrm{~L}$. Importantly, $39.5 \%$ of drinkers reported heavy episodic drinking ${ }^{1}$.

These increasing numbers are noteworthy in part because chronic alcohol use can result in many diseases. The most common disease associated with alcohol is hepatosteatosis, which in the long term increases incidence of hepatocellular carcinoma $(\mathrm{HCC})^{2}$. Although the leading causes of HCC are 
infections with hepatitis B virus (HBV) and hepatitis $\mathrm{C}$ virus, abuse of alcohol, defined as an average daily alcohol consumption of over $80 \mathrm{~g}$, contributes to $15.7 \%$ of liver cancer cases in China ${ }^{3}$. In the United States, which is an area of lower HBV prevalence, it has been reported that $32 \%$ of HCC cases are related to alcohol consumption ${ }^{4}$. As HBV vaccination becomes more prevalent and examination of blood used for transfusions becomes more effective, it can be anticipated that the proportion of HCC related to hepatic viruses will drop, and alcohol will eventually become the leading cause of $\mathrm{HCC}^{5}$. Therefore, understanding the etiology of HCC, especially in the context of alcohol consumption, is important for prevention and treatment.

Interestingly, binge drinking is associated with an accumulation of neutrophils in the liver, and this accumulation is a hallmark of alcohol consumption ${ }^{6}$. In addition, neutrophil extracellular traps (NETs) could contribute to liver injury after binge alcohol use ${ }^{7}$. NETs are networks of extracellular fibers, primarily composed of neutrophil DNA, that form in the presence of exogenous copper ${ }^{8}$. While NETs are important in the removal of pathogens, NETs will sometimes also damage adjacent cells. Studies have shown that inhibition of neutrophils can prevent alcoholic steatohepatitis and HCC that is induced by chemical carcinogens ${ }^{9,10}$. For example, Kolaczkowska et al. showed that damage was decreased significantly when NETs were depleted in the context of bacterial-induced liver injury ${ }^{11}$. Thus, neutrophils and NETs seem to play a critical role in several aspects of liver damage. Whether these factors also contribute to alcoholic hepatosteatosis and alcohol-related HCC remains unclear.

It is well known that alcohol contributes to hepatosteatosis and HCC through direct insult to the liver ${ }^{12}$; however, beyond this direct mechanism, alcohol may also damage the intestinal barrier and induce overgrowth of gut bacteria. As a result, overgrowth of bacteria and overproduction of immunogenic components, including lipopolysaccharide (LPS), would translocate to the liver, potentially contributing to intrahepatic inflammation, which has been proven to be related to steatosis and carcinogenesis ${ }^{13,14}$. Although LPS has been proved to trigger NETs formation in many conditions, binge alcohol suppressed additional LPS-induced hepatic NET formation in mice ${ }^{7}$. Whether intestinal derived LPS is essential for NETs formation in alcoholic hepatosteatosis and subsequent HCC still unsure. According to the evidence presented above, we hypothesize that NETs and LPS play key roles in alcoholic hepatosteatosis and alcohol-induced HCC, and we performed the following studies to test this hypothesis.

\section{Methods}

\section{Collection of human samples}

Serum samples were collected from patients hospitalized in the Liver Disease Center or General Surgery Department at the Second Affiliated Hospital of Xi'an Jiaotong University. The diagnosis of alcoholic liver disease was based on the guideline which issued by Chinese Society of Gastroenterology ${ }^{15}$, and all the HCC patients was diagnosised by histology. Control stool samples were collected from patients diagnosed with inguinal hernia, gallstones, and thyroid nodular goiter and without histories of alcohol 
consumption or any liver disease prior to their surgeries in the surgical clinic at the General Surgery Department, Second Affiliated Hospital of the Xi'an Jiaotong University. All human experiments were reviewed and approved by the Ethics Committee of Second Affiliated Hospital of the Xi'an Jiaotong University (No. 2018-2115).

\section{Animals}

Neutrophil elastase (NE) and Toll-like receptor (TLR) 9 knockout mice (C57BL/6 background) were obtained from The Jackson Laboratory. Mice were maintained under $12 \mathrm{~h}$ light-dark cycles in specificpathogen free (SPF) rooms of the Experimental Animal Center, Xi'an Jiaotong University School of Medicine.

\section{Animal models}

A mouse model of choronic plus binge feeding alcoholic hepatosteatosis was developed by feeding male mice (8 to 10 weeks of age) a Lieber-DeCarli liquid alcohol diet (Trophic Animal Feed High-Tech Co., China) for 4 weeks followed by three gavages of a single dose of ethanol ( $5 \mathrm{~g} / \mathrm{kg}$ body weight per day). Control mice were fed with a Lieber-DeCarli regular control diet and saline. All mice were euthanized at 9 hours after last gavage for sample harvest.

An animal model of alcoholic HCC was also developed. Since HCC does not arise solely through the use of alcohol ${ }^{16}$, mice were also treated with diethylnitrosamine (DEN) (Cool Chemistry, Beijing, China) to induce HCC. Male mice (4 weeks of age) were injected intraperitoneally with DEN $(75 \mathrm{mg} / \mathrm{kg}$ ) once per week from weeks 4 through 6 . From weeks 7 through 9 , the dose was increased to $100 \mathrm{mg} / \mathrm{kg}$ once per week. Mice were fed a 4\% Lieber-DeCarli liquid alcohol diet from weeks 10 to 17 , and they were euthanized for sample harvest following week 17. Control mice were treated with same protocol except without alcohol feeding. LPS (,Sigma, US) was daily administered by Subcutaneous injection $(300 \mu \mathrm{g} / \mathrm{kg} / \mathrm{d})$.

\section{Immunofluorescence}

Fresh tissue was fixed in $4 \%$ paraformaldehyde overnight. Fixed tissues were dehydrated with a series of alcohol solutions, and the tissue was embedded into paraffin blocks. After slices were prepared, paraffin was removed with xylene, and the slice was hydrated with an ethanol gradient. Antigen was unmasked with sodium citrate and a methanol incubation. Non-specific binding sites were blocked with goat serum, and the tissues were incubated with primary antibody overnight at $4^{\circ} \mathrm{C}$. After several washes, samples were incubated with a fluorescent dye-labeled secondary antibody for $1 \mathrm{~h}$, and nuclei were counterstained with DAPI. Slides were mounted with antifade mounting medium.

\section{Oil red 0 staining}

Liver tissues were frozen in Tissue-Tek O.C.T. compound and cut into $10 \mu \mathrm{m}$ sections. Slices were fixed with ice cold $10 \%$ formalin. The slices were washed with $60 \%$ isopropanol and then stained with oil red 0 
solution for $5 \mathrm{~min}$. Following another $60 \%$ isopropanol wash, nuclei were stained with hematoxylin, and slices were mounted.

\section{Quantitative RT-PCR}

Fresh tissues were homogenized in Trizol solution (Sangon Biotech, Shanghai, China), and then RNA was extracted according to the manufacturer's instructions. RNA was diluted with RNA-free water, and DNA was removed with the TURBO DNA-free Kit (ThermoFisher, Waltham, MA, USA) following the manufacturer's protocol. RNA concentrations were determined with a NanoDrop 2000. RNA $(1 \mu \mathrm{g})$ was used to make cDNA with cDNA Synthesis SuperMix (Bioteke). A final qPCR $20 \mu \mathrm{L}$ reaction mixture was made with $1 \mu \mathrm{L}$ cDNA, $10 \mu \mathrm{L} 2 X$ SYBR GREEN MasterMix and water, and qPCR was performed on an Exicycler $^{\text {TM }} 96$ (Bioneer) machine. Primer sequences are shown in Table 1. GAPDH was used as a control gene. Relative mRNA expression was calculated by the $2^{-\triangle \Delta}$ CT method with target gene expression in the control group set as 1 .

Table 1

Characters for the patient with and without ALD

\begin{tabular}{|c|c|c|c|c|}
\hline & ALD $(n=42)$ & $\begin{array}{l}\text { Health control } \\
(n=19)\end{array}$ & $\begin{array}{l}\text { ALD plus HCC } \\
(n=31)\end{array}$ & $\operatorname{HCC}(n=36)$ \\
\hline age & $48.3(35-70)$ & $46.9(32-61)$ & $59.2(51-68)$ & $61.3(53-71)$ \\
\hline Male & 41 & 11 & 31 & 26 \\
\hline Female & 1 & 8 & 0 & 10 \\
\hline BMI & $\begin{array}{l}26.3(18.3- \\
29.6)\end{array}$ & 27.1(20.4-28.6) & $26.7(24.1-30 / 6)$ & $\begin{array}{l}27.4(23.3- \\
30.1)\end{array}$ \\
\hline $\begin{array}{l}\text { Duration of drinking } \\
\text { (years) }\end{array}$ & $23(2-48)$ & $\mathrm{N} / \mathrm{A}$ & $33(10-48)$ & $\mathrm{N} / \mathrm{A}$ \\
\hline Platelets $\left(\times 10^{9} / \mathrm{L}\right)$ & 154(41-302) & $190(125-316)$ & $94(54-179)$ & $103(67-178)$ \\
\hline $\operatorname{ALT}(I U / L)$ & $49(16-253)$ & $22(9-36)$ & $63(27-101)$ & $55(29-121)$ \\
\hline AST (IU/L) & $73(26-358)$ & $27(14-38)$ & $71(40-108)$ & $75(43-121)$ \\
\hline TBIL (umol/L) & $\begin{array}{l}15.7(5.6- \\
81.7)\end{array}$ & $12.1(5.8-15.7)$ & $14.6(7.8-27.4)$ & $\begin{array}{l}13.1(8.4- \\
23.9)\end{array}$ \\
\hline $\operatorname{ALB}(\mathrm{g} / \mathrm{L})$ & $\begin{array}{l}38.1(26.7- \\
49.4)\end{array}$ & $44.8(34.9-50.4)$ & $35.8(30.2-43.7)$ & $\begin{array}{l}38.1(31.4- \\
44.5)\end{array}$ \\
\hline $\operatorname{Scr}(\mu \mathrm{mol} / \mathrm{L})$ & $88(66-133)$ & $91(57-121)$ & $94(70-118)$ & $90(63-127)$ \\
\hline
\end{tabular}

\section{Liver triglyceride (TG) detection}

Fresh liver samples were weighed and homogenized in PBS on ice. The soluble fraction was collected following centrifugation, and the protein was detected with a bicinchoninic acid (BCA) ssay using bovine 
serum albumin as a reference. Samples were diluted with PBS to achieve equal protein concentrations, and TG was quantified with the Triglycerides Assay Kit (Nanjing Jiancheng Bio. Co.). Briefly, $2.5 \mu \mathrm{L}$ of the sample was incubated with $200 \mu \mathrm{L}$ of the working solution for $10 \mathrm{~min}$ at $37^{\circ} \mathrm{C}$, and the absorbance at 510 $\mathrm{nm}$ was determined. TG concentration was determined via comparison to a standard curve.

\section{ELISA}

Liver samples were weighed and homogenized in cell extraction buffer (Wanleibio. Co., China) with protease inhibitors on ice. After samples were vortexed for $30 \mathrm{~min}$, the supernatant was collected with centrifugationat $5000 \mathrm{rpm}$, and the protein content was quantified with a BCA Assay using bovine serum albumin as a reference. Samples were diluted with PBS to achieve equal protein concentrations. First, 96 well plates were coated with coating solution, and plates were then washed with washing buffer. Next, wells were blocked with blocking buffer, and standards and samples $(100 \mu \mathrm{L})$ were added into wells. After an incubation, wells were washed, and the detection antibody solution was added, followed by addition of streptavidin-horseradish peroxidase solution. Next, TMB substrate solution was added, and added stop solution was added after a $30 \mathrm{~min}$ incubation. Absorbances were read at $450 \mathrm{~nm}$. Concentrations were determined based on a standard curve.

\section{Statistical analysis}

SPSS 20.0 software (IBM, US) was used for data analysis. Numerical data are shown as mean \pm standard deviation. Student's t-tests were used to compare differences between groups. $P$ values lower than 0.05 were considered significantly different.

\section{Results}

\section{NETs and inflammation increased in human ALD and alcoholic HCC}

Samples were collected from a total of 42 patients with alcoholic liver disease (ALD). The characters of patients shows in Table 1.Based on our investigation, ALD patients had a higher serum concentration of myeloperoxidase (MPO)-DNA complexes than did normal control patients $(P<0.05)$ (Fig. 1A). Meanwhile serum concentrations of lipopolysaccharide (LPS) and IL-6 were also higher in ALD patients (Fig. 1B, C). Serum samples from 36 HCC patients without ALD and 31 patients with ALD and HCC patients were collected. The characters of patients shows in Table 1. The serum concentration of MPO-DNA was significantly higher in ALD plus HCC patients than in HCC patients without ALD $(P<0.05)$, and LPS and IL6 concentrations were significantly higher in ALD/HCC patients $(P<0.05)$ (Fig. 1A-C).

\section{Alcohol increases formation of intrahepatic NETs}

Neutrophil infiltration is a typical pathologic feature of human alcoholic liver disease ${ }^{17}$, and it could also be seen in a chronic-binge murine model of alcohol consumption ${ }^{18}$. Here, we found that chronic-binge 
alcohol consumption increased serum MPO-DNA in both alcoholic hepatosteatosis and alcoholic HCC models (Fig. 1D). We then investigated levels of other markers of NET formation, citrullinated histone H3 (cit H3) and NE, by immunofluorescence. This assay indicated more levels of fluorescence in mice of both the alcoholic hepatosteatosis and HCC models as compared to paired mice fed control diets (Fig. 1E, F). The strength of fluorescence was also significantly increased in alcohol treatment groups relative to pair feeding groups $(P<0.05)$ (Fig. 1E, F).

\section{NETs promote alcohol-induced hepatosteatosis and alcohol-related HCC.}

Since NE plays a critical role in lysing of neutrophil membranes in the formation of NETs, NETs do not form in NE KO mice. These mice, then, provided an important model to test the impact of NETs on development of alcohol-related disorders. After 5 weeks of alcohol consumption, positive regions stained by oil red, which stains intrahepatic fat, were significantly less in NE KO mice than in WT mice treated similarly (Fig. 2A). Serum TG increased in binge alcohol-treated mice, but this increase in serum TG was attenuated in NE KO mice (Fig. 2B). Serum ALT was elevated significantly in alcohol-fed mice as compared to paired controls, and this increase was attenuated in in NE KO mice $(P<0.05)$ (Fig. 2C). Similarly, while liver mRNA expression of the inflammation markers tumor necrosis factor (TNF)-a, IL-6, cyclin B1, CXCL-2 and chemokine ligand 2 (CCL2) were increased upon binge alcohol administration for 5 weeks, these increases were significantly lower in NE KO mice $(P<0.05)$ (Fig. 2D).

In the murine model of alcoholic HCC, some tumors were formed in WT mice, but few tumors were seen in NE KO mice. Both average tumor number and maximum tumor size were significantly higher in WT mice relative to NE KO mice $(P<0.05)$ (Fig. 2E). Meanwhile, serum ALT concentrations were decreased in NE $\mathrm{KO}$ mice as compared to WT mice (Fig. 2F). We further tested the liver mRNA levels of CCL-2, cyclin B1, cyclin B2, IL-6, TNF-a and proliferating cell nuclear antigen (PCNA), and results showed that mRNA expression of all of these genes was lower in NE KO mice than WT mice $(P<0.05)$ (Fig. 2G).

\section{Intestinal bacteria and LPS promotes NETs formation in the liver upon chronic alcohol consumption}

After we confirmed that alcohol stimulated the formation of intrahepatic NETs and further relate this increase to alcohol-induced hepatosteatosis and HCC, we endeavored to figure out an upstream inducer of NET formation. Since the gut-liver axis has been shown to play a critical role in many liver diseases, we considered that some product of intestinal bacteria could be the key factor. When we depleted gut bacteria with a cocktail of antibiotics in mice of the alcohol-induced hepatosteatosis model, we found that intrahepatic LPS was significantly decreased relative to model mice without the cocktail treatment $(P$ $<0.05$ ) (Fig. 3A) In addition, upon antibiotic treatment, fewer cells staining positive for intrahepatic NE and cit H3 were observed (Fig. 3B, C), and serum MPO-DNA was significantly decreased (Fig. 3D), indicating lower NET formation.

When mice were treated with both antibiotics and LPS, although the bacterial population of stool was significantly reduced, both stool and liver LPS levels were increased (data not shown). After a 5 weeks 
binge alcohol treatment, serum MPO-DNA was recovered with the combined treatment, and intrahepatic $\mathrm{NE}$ and cit H3-positive cells also increased relative to mice treated only with antibiotics (Fig. 3B, C). Similarly, serum MPO-DNA was also significantly recovered upon oral treatment with LPS (Fig. 3D).

Similar results were also seen in the alcoholic HCC model. Depletion of intestinal bacteria by a cocktail of antibiotics attenuated intrahepatic LPS (Fig. 3E), and serum MPO-DNA and intrahepatic cells positive for $\mathrm{NE}$ and cit $\mathrm{H} 3$ also significantly decreased (Fig. 3F-H). On the other hand, in this model, antibiotics did not attenuate serum MPO-DNA level or number of NE and cit H3-positive cells when mice were also treated with LPS (Fig. 3F-H).

\section{LPS plays a role in alcoholic hepatosteatosis and alcohol- related HCC}

As we have shown that LPS plays a role in the alcohol-induced formation of intrahepatic NETs models of in alcoholic hepatosteatosis and alcoholic HCC, and as NETs are known to correlate with these diseases, we predicted that LPS plays a role in these diseases. Accordingly, in the model of alcohol-induced hepatosteatosis, antibiotics decreased the ballooning degeneration of hepatocytes and inflammation of intrahepatic cells as demonstrated by hematoxylin and eosin (HE) staining, while these phenomena increased when antibiotic treatment was combined with oral LPS administration. Similarly, upon staining with oil red, less red staining was observed in mice treated with antibiotics, but more staining was observed when antibiotics were combined with LPS (Fig. 4A). Next, we determined concentrations of TG in the liver and found that it was decreased in the antibiotics group but was recovered in the group treated with antibiotics and LPS (Fig. 4B). Serum ALT levels were much lower in the antibiotics group and were increased with LPS (Fig. 4C). Meanwhile, liver expression of the mRNAs of TNF-a, IL-6, CCL2, C-X-C motif ligand 1 (CXCL1), cyclin B1 and IL-6 were significantly decreased after treatment with antibiotics, while this effect was attenuated upon treatment with LPS $(P<0.05)$ (Fig. 4D).

In the alcohol-related HCC model, the use of antibiotics attenuated HCC carcinogenesis. The number of tumors and the maximum tumor size were significantly reduced in mice administered antibiotics, while the number and size increased upon combining of antibiotics with LPS treatment (Fig. 4E). At the time of sacrifice, serum ALT concentrations were significantly lower in antibiotic-treated mice than in mice not treated with antibiotics, and these concentrations were recovered upon co-administration of LPS (Fig. 4F). Similarly, liver expression of the mRNA of CCL-2, cyclin B1, cyclin B2, TNF-a, IL-6, and PCNA was significantly decreased upon treatment of mice with antibiotics, and expression of these genes was increased again upon co-treatment of mice with antibiotics and LPS (Fig. 4G).

\section{Lack of TLR4 decrease NETs formation in chronical alcohol consumption}

After confirming that intestinal derived LPS promotes the formation of NETs during chronic alcohol consumption, we considered that TLR4, which is LPS receptor, may also play a role in the formation of NETs. A strain of TLR4 KO mice was used in the study. There was no difference in the concentration of 
intrahepatic LPS between the mice with and without TLR4 in the model of alcoholic steatosis (data not shown). However, when we examined expression of NE and cit $\mathrm{H} 3$ in the liver by immunofluorescence, we observed fewer positive cells in TLR4 KO mice than in WT mice (Fig. 5A, B). The serum MPO-DNA level decreased significantly in the absence of TLR4, as well (Fig. 5C).

The same pattern was observed in the alcohol-related HCC model. The intrahepatic LPS was constant upon depletion of TLR4, but fewer cells were stained positive for NE and cit H3 in TLR4 KO mice than in WT mice (Fig. 5D, E). Similarly, the serum MPO-DNA concentration was reduced significantly in TLR4 KO mice relative to $\mathrm{WT}$ mice (Fig. 5F).

\section{Lack of TLR4 decreases formation of NETs and HCC in the alcohol-induced HCC model}

Finally, we investigated whether TLR4 also plays a role in alcoholic steatosis and alcohol-induced HCC. In the murine model of alcoholic steatosis, fewer ballooning degeneration hepatocytes and intrahepatic inflammation cells could be seen in the liver by HE staining. Intrahepatic fat deposition, as revealed by oil red staining, was less in TLR4 KO mice than in WT mice (Fig. 6A). Serum ALT and TG concentrations were much decreased in TLR4 KO mice (Fig. 6B-C). Liver expression of CXCL1, CXCL2, CCL2, IL-6, TNF-a and cyclin B1 mRNA was higher in WT mice than in TLR4 KO mice (Fig. 6D).

In the murine model of alcoholic HCC, some tumors had occurred by the time of harvest, but few tumors were observed in TLR4 KO mice, and TLR4 KO mice had significantly decreases in both the average number of tumors and the size of largest tumor (Fig. 6E). Upon euthanizing, serum levels of ALT were found to be significantly decreased in TLR4 KO relative to WT mice (Fig. 6F). Meanwhile, mRNA expression of PCNA, CCL2, TNF-a, IL-6, cyclin B1 and cyclin B2 were decreased in TLR4 KO mice as compared to WT mice(Fig. 6G).

\section{Discussion}

Binge drinking is a global problem, and it is well known that a potential consequence of binge drinking is hepatic steatosis, which can lead to HCC. In fact, many clinical studies have shown that chronic alcohol consumption is an independent risk factor for $\mathrm{HCC}^{4,19}$, and the WHO lists alcohol as a risk factor for HCC occurrence $^{1}$. In animal studies, although HCC does not occur only with long-term alcohol administration, alcohol consumption significantly accelerates the genesis of HCC and increases tumor load when combined with a chemical inducer ${ }^{20}$.

Intrahepatic neutrophil infiltration is a hallmark of binge alcohol consumption, and neutrophils have been proven to play an important role in the occurrence of $\mathrm{HCC}^{9}$. Neutrophils are also believed to be the major source of many cytokines, including matrix metalloproteinase (MMP)-9, IL-10 and CCL2, which are thought to play roles in enhanced tumorigenesis, invasion and growth. A strong infiltration of neutrophils into the liver is also believed to be independently associated with poorer survival in human $\mathrm{HCC}^{21}$. It has 
been reported that neutrophils contribute to $A \mathrm{LD}^{22}$ and that antibody-mediated depletion of hepatic neutrophils will attenuate $\mathrm{HCC}^{9}$. However, the mechanisms behind the involvement of neutrophils in HCC has not been fully revealed. In particular, NETs are a neutrophil-formed structure that have been proven to form in nonalcoholic steatohepatitis and HCC and to be involved with metastasis of HCC and with many other liver diseases ${ }^{23,24}$. However, as in the case of neutrophils, the precise role of NETs in alcohol-related $\mathrm{HCC}$ has not been revealed.

According to our initial data involving human subjects, we found that ALD and alcohol-related HCC correlated with increased serum levels of MPO-DNA complexes, which are indicators of the formation of NETs. However, without liver biopsy samples, we could not confirm whether NETs were formed in the liver or in extrahepatic organs, so a murine study was necessary. With mice chronic alcohol feeding models, we found an abundance of NETs formed in the liver under conditions of chronic alcohol consumption.

The process of NETosis, which is a form of programmed cell death, and the elimination of antigens by NETs, an abundance of reactive oxygen species (ROS), cytokines and enzymes are released ${ }^{25}$. This release would not only remove the pathogen but would also impair the surrounding healthy cells ${ }^{25}$. Kolaczkowska et al. found that intrahepatic neutrophil infiltration is critical for bacterial-induced liver injury, and liver injury decreased $80 \%$ when NET formation was inhibited in the liver ${ }^{11}$. Similarly, NETs have been found to contribute to liver injury associated with sepsis that is induced by binge alcohol consumption 7 , along with many other liver disorders not associated with alcohol, such as non-alcoholic steatohepatitis, ischemia reperfusion injury and portal hypertension ${ }^{25-27}$.

However, whether NET formation is a risk factor for alcohol-induced HCC remains unproven. In this study, a NE KO mice, in which neutrophils fail lyse and form NETs, were used. Using this model, we found that alcoholic hepatosteatosis together with many inflammation markers were significantly attenuate upon loss of NET formation. It is well known that chronic inflammation relate to carcinogenesis, and hepatosteatosis is also known as a risk factor for HCC. So there is no surprise that the absence of NETs delays HCC occurrence.

While we confirmed that NETs are related to alcoholic hepatosteatosis and $\mathrm{HCC}$, the reason for intrahepatic accumulation of NETs upon alcohol consumption had still not been fully revealed. Notably, alcohol binge consumption not only insults the liver but also affect the intestinal tract. Alcohol and its metabolites can injure the intestinal mucosa directly and contribute to intestinal inflammation, which result in an increasing of gut permeability ${ }^{28}$. In addition, consuming alcohol can also slow intestinal movement and result in overgrowth of intestinal bacteria ${ }^{29,30}$. Together, these factors lead to the occurrence of leaky gut and a movement of bacteria and bacterial products into the liver via the portal vein $^{29,30}$. The role of the gut-liver axis has been implicated in many liver diseases, including some alcohol-related liver diseases, and LPS has been shown to be a mediator of the communication between the liver and the gut ${ }^{31,32}$. However, with leaky gut in alcohol consumption, there remains some additional bacterial products that might be involved in intrahepatic translocation, including LPS. 
An important task of innate immune system is to eliminate bacteria and their metabolites rapidly from the circulation. In the mice sepsis model, most bacteria are sealed up or trapped in liver immediately once

they are injected into blood. Subsequently, neutrophile granulocytes are recruited to the liver soon ${ }^{33,34}$. In this study, it is found by combined removal of intestinal bacteria that intestinal bacteria are the causes of NETs under the occurrence of alcoholic steatohepatitis and subsequent HCC. However, existing intestinal sterilization methods can eliminate various intestinal bacteria and relevant components (e.g. LPS, LTA). Rare studies have discovered the relationship between LTA and NET ${ }^{35}$, and some studies even demonstrated that LTA had protective effect on NETs-induced side damages ${ }^{36,37}$. Many experiments have proved that LPS, a PAMPs of G-bacteria, can promote adhesion of neutrophile granulocytes in hepatic sinusoid and promote NETs formation through its receptor TLR4 ${ }^{37,38}$. It is also proved that LPSTLR4 pathway participates in progresses of NASH and HCC and NETs are also proved to be related with chemical induced $\mathrm{HCC}^{23,39,40}$. Based on removal of mice intestinal bacteria, LPS was administrated and results showed that formation of NETs is recovered by supplementing LPS. Meanwhile, formation of NETs is inhibited after TLR4 missing of mice, indicating that intestinal LPS is the key factor of NETs formation during alcohol intake and thereby causes alcoholic hepatosteatosis and subsequent HCC.

However, TLR4 also can stimulate NETs formation through non-bacteria factors. For instance, platelet of patients with AAV can promote in vitro formation of NETs through TLR4 without assistance of bacteria ${ }^{41}$. HMGB also causes adjacent damages by promoting formation of NETs through TLR4 in liver ischemiareperfusion injury ${ }^{42}$. It is found in the study that activation of LPS-TLR4 pathway involves in cell damages and ROS releasing at inhalation of alcohol, thus releasing several DAMPs ${ }^{43}$. Although this study found based on a TLR4 KO mice test that TLR4 participates in formation of NETs at inhalation of alcohol and thereby induces alcoholic hepatosteatosis and subsequent HCC, it remains unknown whether TLR4 induces hepatosteatosis and subsequent HCC through direct or indirect pathway or their collaboration. These will be our further research directions.

\section{Conclusions}

In conclusion, this study proved that intestinal derived LPS stimulates NET formation via the TLR4 pathway and increases intrahepatic inflammation in chronic alcohol consumption, which in turn increases the genesis of alcoholic hepatosteatosis and subsequent HCC.

\section{Declarations}

Ethics approval and consent to participate: All human sample collections were reviewed and approved by the Ethics Committee of Second Affiliated Hospital of the Xi'an Jiaotong University (No. 2018-2115).

Consent for publication: Not applicable

Availability of data and material: The datasets used or analyzed during the current study are available from the corresponding author on reasonable request. 
Competing interests: The authors declare no competing interests.

Funding: This work was supported by grants from the National Natural Science Foundation of China (Grant No. 81602401) and Natural Science Foundation of Shaanxi Province (2020JM-404) to Y.L. The work was also supported by the National Natural Science Foundation of China (Grant No.82003998) to S.C. and (Grant No. 81902449) M.X.

Authors' contributions: Y.L.: Designed research, conducted experiments, acquired data, analyzed data, wrote the manuscript. S.C., J.W.: Conducted experiments, acquired data, analyzed data, and wrote the manuscript. X.Z.: Designed research, conducted experiments, analyzed data. S.Y., Y.L, M.X., W.Q, H.A., H.L., T.S., J.W.: Acquired data, conducted experiments. G.C.: Designed research, analyzed data, revised the manuscript. All authors revised and approved the manuscript for publication.

Acknowledgements: Not applicable

List of Abbreviations: hepatocellular carcinoma (HCC); neutrophil extracellular traps (NETs); neutrophil elastase (NE); lipopolysaccharide (LPS); Toll-like receptor (TLR); specific-pathogen-free (SPF); diethylnitrosamine (DEN); triglyceride (TG); bicinchoninic acid (BCA); body mass index (BMI); alanine aminotransferase (ALT); myeloperoxidase (MPO); lipopolysaccharide (LPS); histone H3 (cit H3); chemokine ligand 2 (CCL2); tumor necrosis factor-a (TNF-a); proliferating cell nuclear antigen (PCNA); hematoxylin and eosin (HE); C-X-C motif ligand 1 (CXCL1); oxygen species (ROS); pathogen-associated molecular patterns (PAMPs).

\section{References}

1. Organization WH. Global status report on alcohol and health 2018: World Health Organization, 2018.

2. Pekow JR, Bhan AK, Zheng $\mathrm{H}$, et al. Hepatic steatosis is associated with increased frequency of hepatocellular carcinoma in patients with hepatitis C-related cirrhosis. Cancer 2007;109:2490-6.

3. Fan JH, Wang JB, Jiang $\mathrm{Y}$, et al. Attributable causes of liver cancer mortality and incidence in china. Asian Pac J Cancer Prev 2013;14:7251-6.

4. Hassan MM, Hwang LY, Hatten CJ, et al. Risk factors for hepatocellular carcinoma: synergism of alcohol with viral hepatitis and diabetes mellitus. Hepatology 2002;36:1206-13.

5. McGlynn KA, London WT. The global epidemiology of hepatocellular carcinoma: present and future. Clin Liver Dis 2011;15:223-43, vii-x.

6. Wieser V, Tymoszuk P, Adolph TE, et al. Lipocalin 2 drives neutrophilic inflammation in alcoholic liver disease. J Hepatol 2016;64:872-80. 
7. Bukong TN, Cho Y, Iracheta-Vellve A, et al. Abnormal neutrophil traps and impaired efferocytosis contribute to liver injury and sepsis severity after binge alcohol use. J Hepatol 2018;69:1145-1154.

8. Santocki M, Kolaczkowska E. On Neutrophil Extracellular Trap (NET) Removal: What We Know Thus Far and Why So Little. Cells 2020;9.

9. Wilson CL, Jurk D, Fullard N, et al. NFkappaB1 is a suppressor of neutrophil-driven hepatocellular carcinoma. Nat Commun 2015;6:6818.

10. Zhou Z, Xu MJ, Cai Y, et al. Neutrophil-Hepatic Stellate Cell Interactions Promote Fibrosis in Experimental Steatohepatitis. Cell Mol Gastroenterol Hepatol 2018;5:399-413.

11. Kolaczkowska E, Jenne CN, Surewaard BG, et al. Molecular mechanisms of NET formation and degradation revealed by intravital imaging in the liver vasculature. Nat Commun 2015;6:6673.

12. Seitz HK, Bataller R, Cortez-Pinto H, et al. Alcoholic liver disease. Nat Rev Dis Primers 2018;4:16.

13. Leitao HS, Doblas S, Garteiser P, et al. Hepatic Fibrosis, Inflammation, and Steatosis: Influence on the MR Viscoelastic and Diffusion Parameters in Patients with Chronic Liver Disease. Radiology 2017;283:98-107.

14. Gonda TA, Tu S, Wang TC. Chronic inflammation, the tumor microenvironment and carcinogenesis. Cell Cycle 2009;8:2005-13.

15. Li YM, Fan JG, National Workshop on Fatty L, et al. Guidelines of prevention and treatment for alcoholic liver disease (2018, China). J Dig Dis 2019;20:174-180.

16. Machida $\mathrm{K}$, Tsukamoto $\mathrm{H}$, Mkrtchyan $\mathrm{H}$, et al. Toll-like receptor 4 mediates synergism between alcohol and HCV in hepatic oncogenesis involving stem cell marker Nanog. Proc Natl Acad Sci U S A 2009;106:1548-53.

17. Celli R, Zhang X. Pathology of Alcoholic Liver Disease. J Clin Transl Hepatol 2014;2:103-9.

18. Bertola A, Park O, Gao B. Chronic plus binge ethanol feeding synergistically induces neutrophil infiltration and liver injury in mice: a critical role for E-selectin. Hepatology 2013;58:1814-23.

19. Li Y, Yang H, Cao J. Association between alcohol consumption and cancers in the Chinese population-a systematic review and meta-analysis. PLoS One 2011;6:e18776.

20. Brandon-Warner E, Walling TL, Schrum LW, et al. Chronic ethanol feeding accelerates hepatocellular carcinoma progression in a sex-dependent manner in a mouse model of hepatocarcinogenesis. Alcohol Clin Exp Res 2012;36:641-53.

21. Margetts J, Ogle LF, Chan SL, et al. Neutrophils: driving progression and poor prognosis in hepatocellular carcinoma? Br J Cancer 2018;118:248-257. 
22. Lowe PP, Gyongyosi B, Satishchandran A, et al. Alcohol-related changes in the intestinal microbiome influence neutrophil infiltration, inflammation and steatosis in early alcoholic hepatitis in mice. PLoS One 2017;12:e0174544.

23. van der Windt DJ, Sud V, Zhang H, et al. Neutrophil extracellular traps promote inflammation and development of hepatocellular carcinoma in nonalcoholic steatohepatitis. Hepatology 2018;68:13471360.

24. Yang LY, Luo Q, Lu L, et al. Increased neutrophil extracellular traps promote metastasis potential of hepatocellular carcinoma via provoking tumorous inflammatory response. J Hematol Oncol 2020;13:3.

25. Papayannopoulos V. Neutrophil extracellular traps in immunity and disease. Nat Rev Immunol 2018;18:134-147.

26. Du J, Zhang J, Chen X, et al. Neutrophil extracellular traps induced by pro-inflammatory cytokines enhance procoagulant activity in NASH patients. Clin Res Hepatol Gastroenterol 2021:101697.

27. Zhang S, Zhang Q, Wang F, et al. Hydroxychloroquine inhibiting neutrophil extracellular trap formation alleviates hepatic ischemia/reperfusion injury by blocking TLR9 in mice. Clin Immunol 2020;216:108461.

28. Meroni M, Longo M, Dongiovanni P. Alcohol or Gut Microbiota: Who Is the Guilty? Int J Mol Sci 2019;20.

29. Llorente $C$, Jepsen $P$, Inamine $T$, et al. Gastric acid suppression promotes alcoholic liver disease by inducing overgrowth of intestinal Enterococcus. Nat Commun 2017;8:837.

30. Bode C, Bode JC. Effect of alcohol consumption on the gut. Best Pract Res Clin Gastroenterol 2003;17:575-92.

31. Bala S, Csak T, Kodys K, et al. Alcohol-induced miR-155 and HDAC11 inhibit negative regulators of the TLR4 pathway and lead to increased LPS responsiveness of Kupffer cells in alcoholic liver disease. J Leukoc Biol 2017;102:487-498.

32. Ohtani N, Kawada N. Role of the Gut-Liver Axis in Liver Inflammation, Fibrosis, and Cancer: A Special Focus on the Gut Microbiota Relationship. Hepatol Commun 2019;3:456-470.

33. Yan J, Li S, Li S. The role of the liver in sepsis. Int Rev Immunol 2014;33:498-510.

34. Surewaard BG, Deniset JF, Zemp FJ, et al. Identification and treatment of the Staphylococcus aureus reservoir in vivo. J Exp Med 2016;213:1141-51.

35. Wartha F, Beiter K, Albiger B, et al. Capsule and D-alanylated lipoteichoic acids protect Streptococcus pneumoniae against neutrophil extracellular traps. Cell Microbiol 2007;9:1162-71. 
36. McDonald B, Jenne CN, Zhuo L, et al. Kupffer cells and activation of endothelial TLR4 coordinate neutrophil adhesion within liver sinusoids during endotoxemia. Am J Physiol Gastrointest Liver Physiol 2013;305:G797-806.

37. Arroyo R, Khan MA, Echaide M, et al. SP-D attenuates LPS-induced formation of human neutrophil extracellular traps (NETs), protecting pulmonary surfactant inactivation by NETs. Commun Biol 2019;2:470.

38. Munir S, Basu A, Maity P, et al. TLR4-dependent shaping of the wound site by MSCs accelerates wound healing. EMBO Rep 2020;21:e48777.

39. Carpino G, Del Ben M, Pastori D, et al. Increased Liver Localization of Lipopolysaccharides in Human and Experimental NAFLD. Hepatology 2020;72:470-485.

40. Toffanin S, Cornella $\mathrm{H}$, Harrington A, et al. HCC Is promoted by bacterial translocation and TLR-4 signaling: a new paradigm for chemoprevention and management. Hepatology 2012;56:1998-2000.

41. Matsumoto $\mathrm{K}$, Yasuoka H, Yoshimoto $\mathrm{K}$, et al. Platelet CXCL4 mediates neutrophil extracellular traps formation in ANCA-associated vasculitis. Sci Rep 2021;11:222.

42. Huang $\mathrm{H}$, Tohme $\mathrm{S}$, Al-Khafaji $\mathrm{AB}$, et al. Damage-associated molecular pattern-activated neutrophil extracellular trap exacerbates sterile inflammatory liver injury. Hepatology 2015;62:600-14.

43. Guo J, Friedman SL. Toll-like receptor 4 signaling in liver injury and hepatic fibrogenesis. Fibrogenesis Tissue Repair 2010;3:21.

\section{Figures}


Figure 1.

A
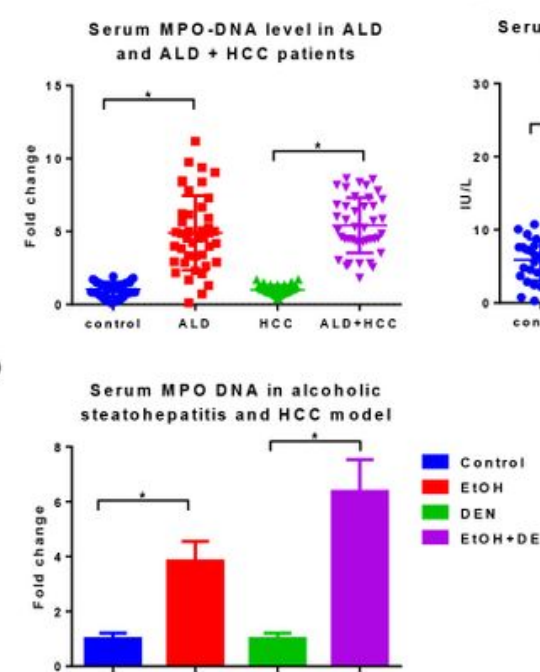

E Cell fluorescence of $\mathrm{Cit} \mathrm{H}_{3}$ in alcoholic
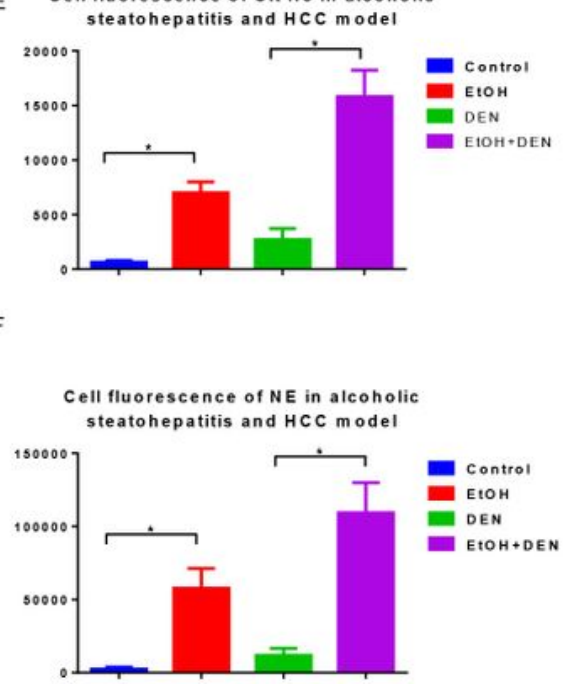

B

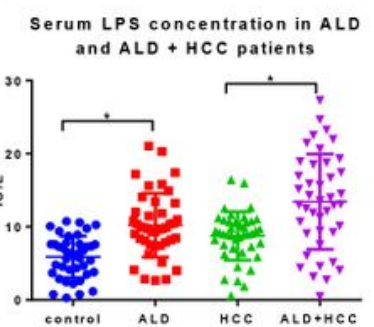

Cit H3

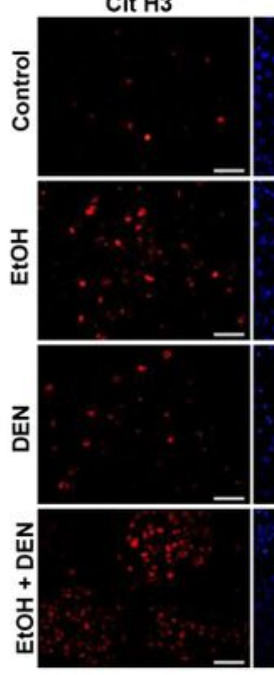

NE

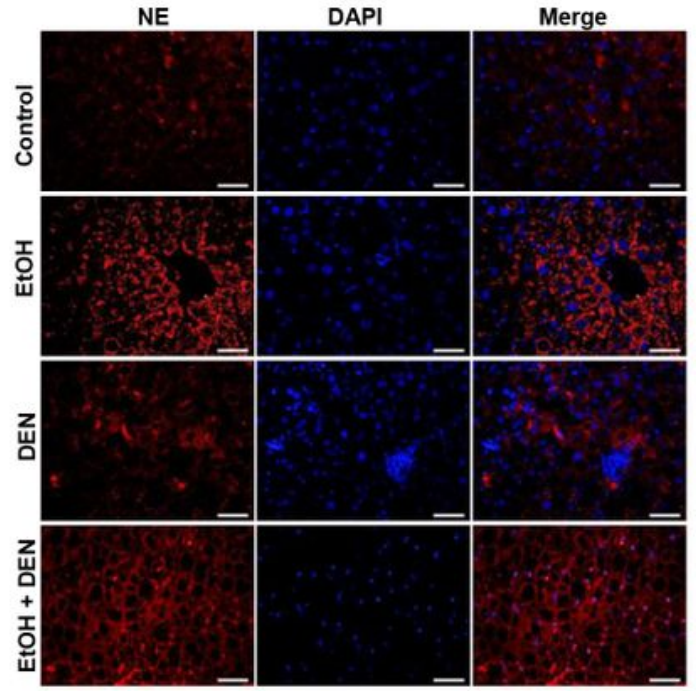

c

Serum IL-6 concentration in ALD and $A L D+H C C$ patients

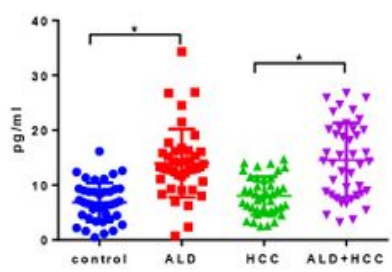

DAPI

Merge

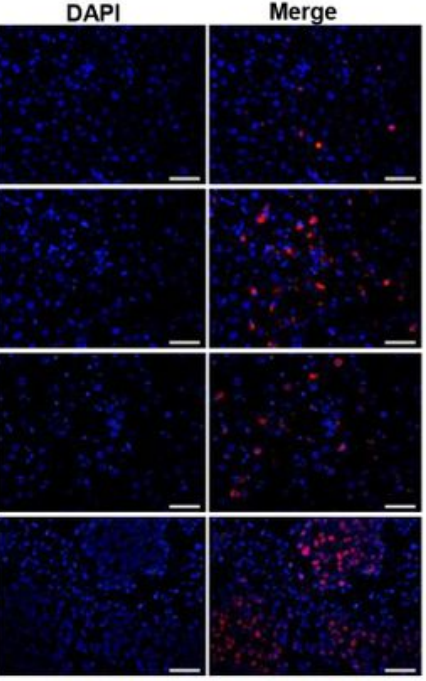

DAPI 
Figure 2 .
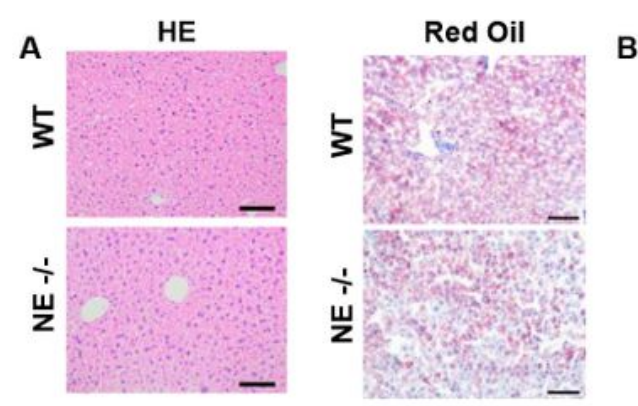

B
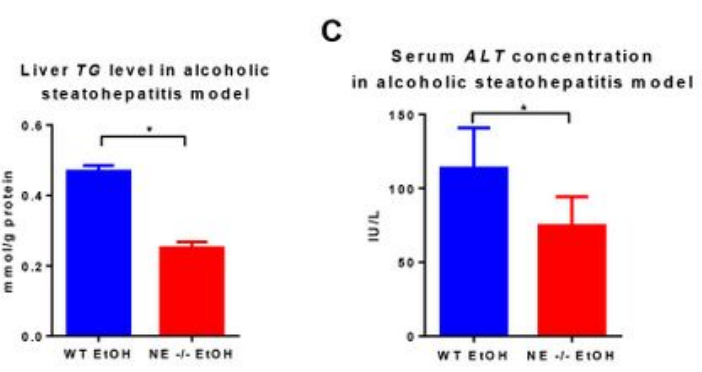

D Liver TNF.a mRNA level in alcoholic steatohepatitis mode

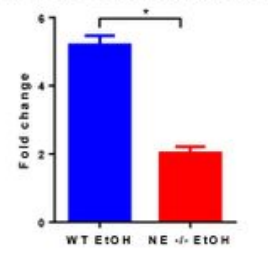

Liver $16.6 \mathrm{mRNA}$ level in
alcoholic steatohepatitis mode

Liver Cyclin B1 mRNA level Liver Cel.2 mRNA level in
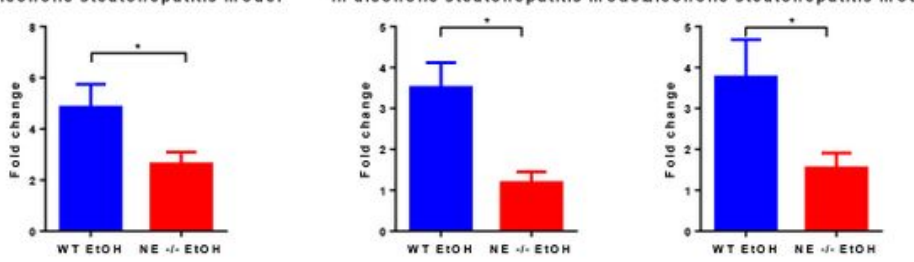

E
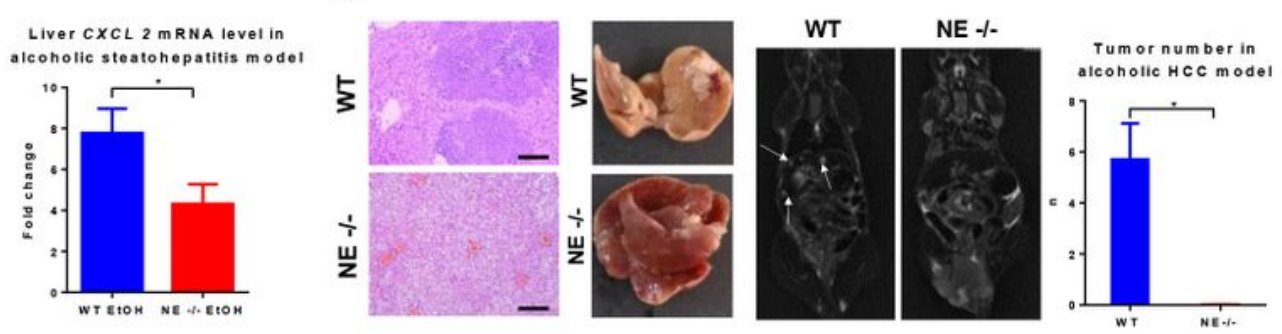

F

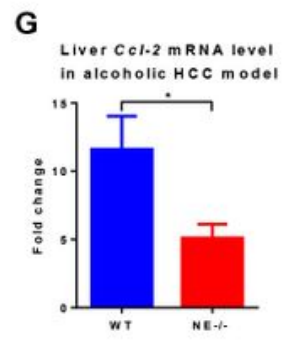

Liver Cyclin B1 mRNA

Serum ALT concentration in alcoholic HCC model Largest tumor in
alcoholic HCC model
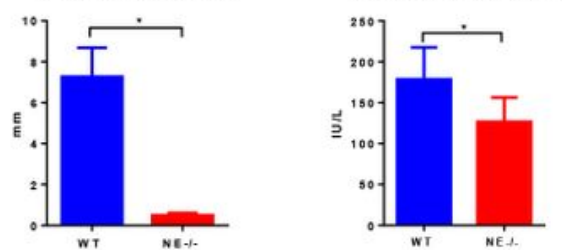
level in alcoholic HCC model

Liver Cyclin B 2 mRNA level in alcoholic HCC model
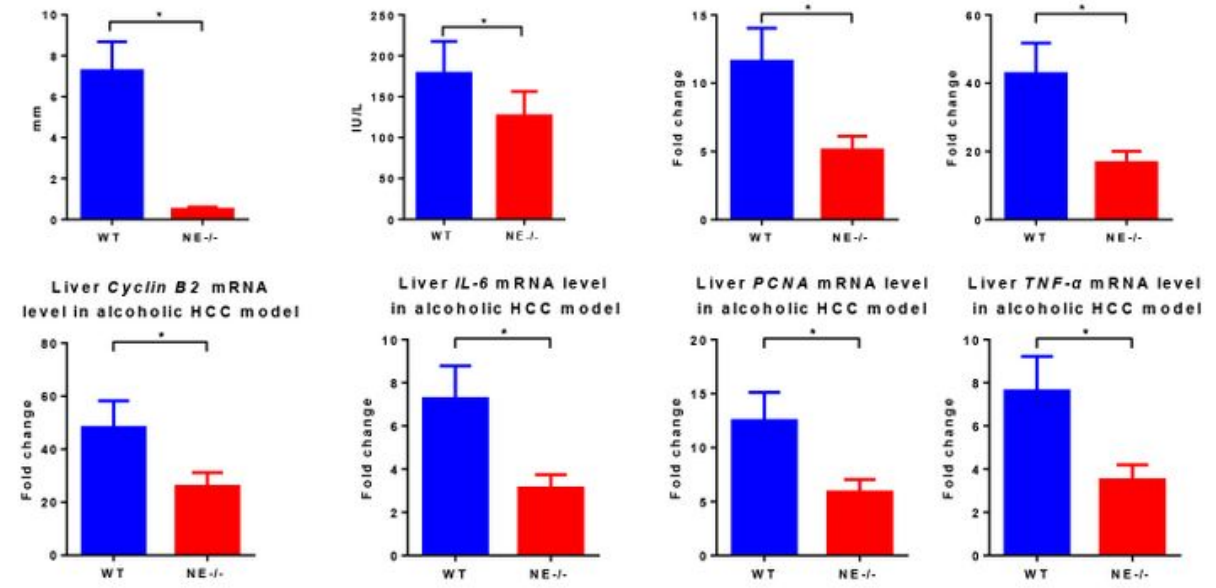

\section{Figure 2}

Lack of NETs leads to alleviation of alcoholic hepatosteatosis and subsequent HCC in mice. A: Mouse liver tissues were visualized by HE staining and oil red staining after 5 weeks of alcohol treatment. B-C: TG and serum ALT levels from livers of alcoholic hepatosteatosis model mice were determined by ELISA. D: WT and NE KO mice underwent alcohol treatment for 5 weeks, and liver levels of TNF-a, IL-6, cyclin B1 CXCL-2 and CCL-2 genes were determined by qPCR. E: Liver tumors from alcoholic HCC model mice were 
analyzed by HE staining, photography and MRI scanning. F: Serum ALT levels were determined by ELISA in models of alcoholic HCC. G: WT and NE KO mice underwent the procedure to develop the alcoholic HCC model for 13 weeks, whereupon liver levels of TNF-a, IL-6, cyclin B1, cyclin B2, CCL-2 and PCNA genes were determined by qPCR.

Figure 3.

A

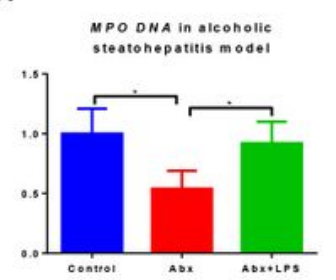

B

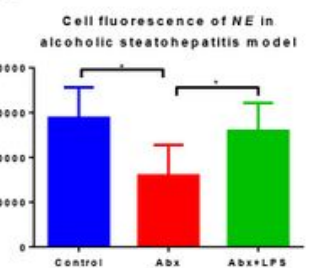

C

Cell fluorescence of $\mathrm{CH}^{\mathrm{H}} \mathrm{H}$ in
alcohollic steatohepatitis model

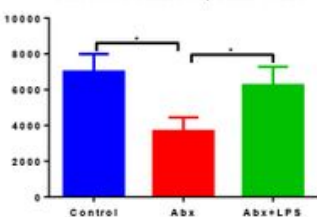

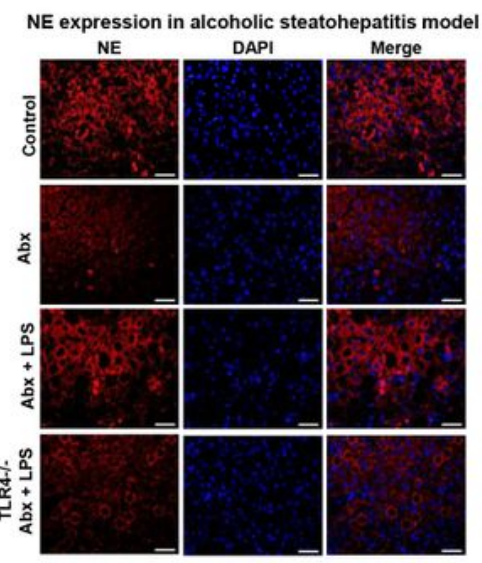
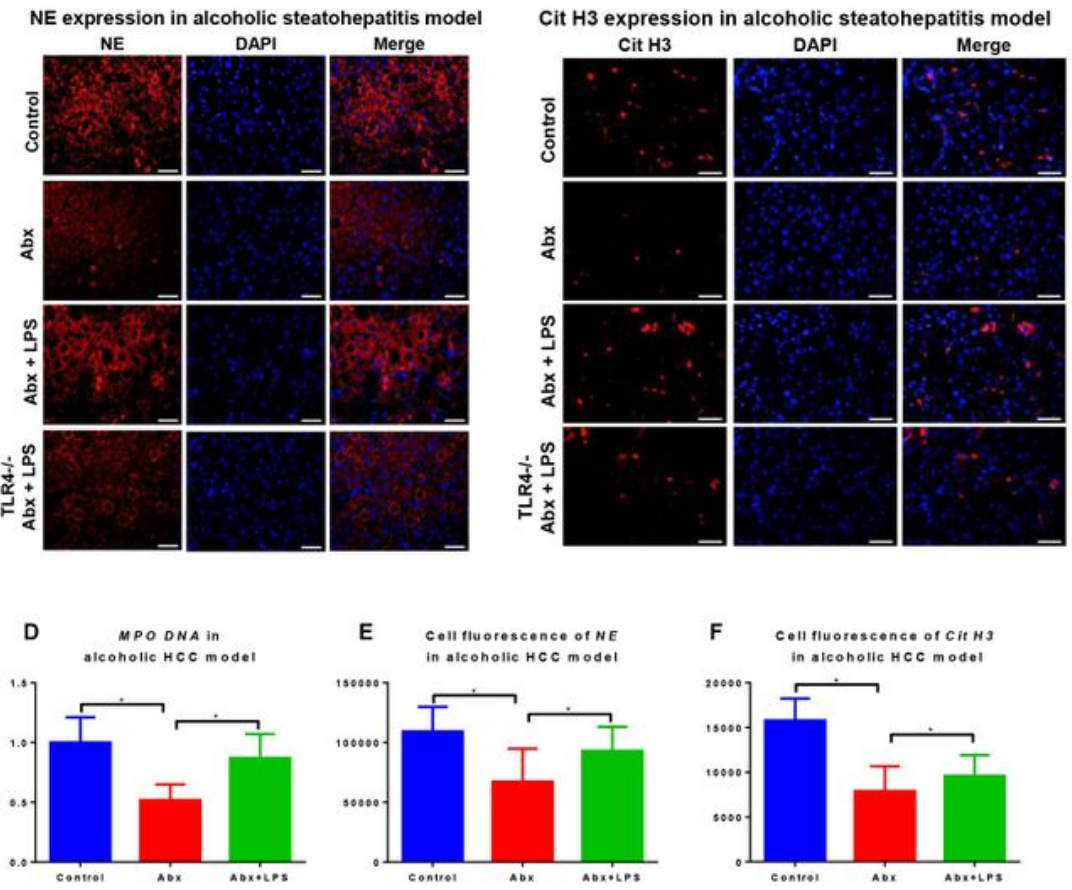

NE expression in alcohol induced $\mathrm{HCC}$ model

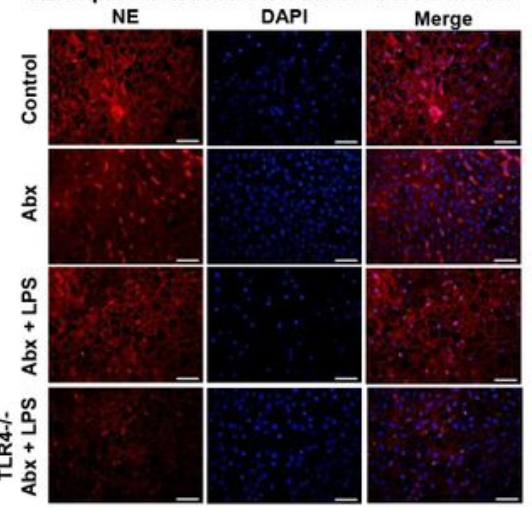

Cit $\mathrm{H} 3$ expression in alcohol induced $\mathrm{HCC}$ model

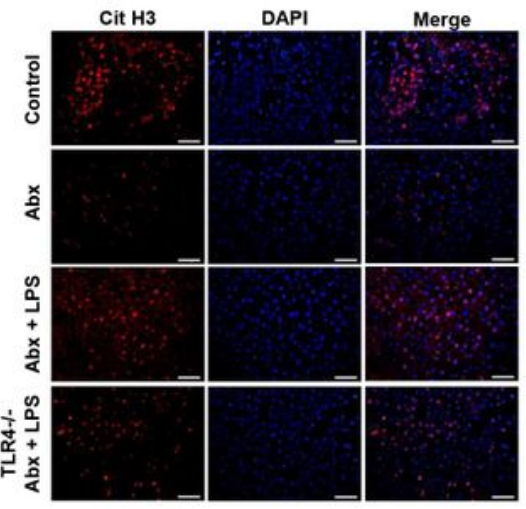

Figure 3 
Gut-derived LPS promotes intrahepatic NET formation during mice alcoholic hepatosteatosis and subsequent HCC. A: Liver LPS was determined by ELISA in the mouse model of alcoholic hepatosteatosis. B-C: Alcoholic hepatosteatosis livers were tested for NETs by the expression of cit H3 and NE, which were determined by immunofluorescence. D: Alcoholic hepatosteatosis liver MPO-DNA was determined by ELISA in the mouse model of alcoholic hepatosteatosis. E: Liver LPS was determined by ELISA in the mouse model of alcoholic HCC. F-G: Alcoholic HCC livers were tested for NETs by expression of cit $\mathrm{H} 3$ and NE, which were determined by immunofluorescence. $\mathrm{H}$ : Liver MPO-DNA was determined by ELISA in the mouse model of alcoholic HCC. 
Figure 4.
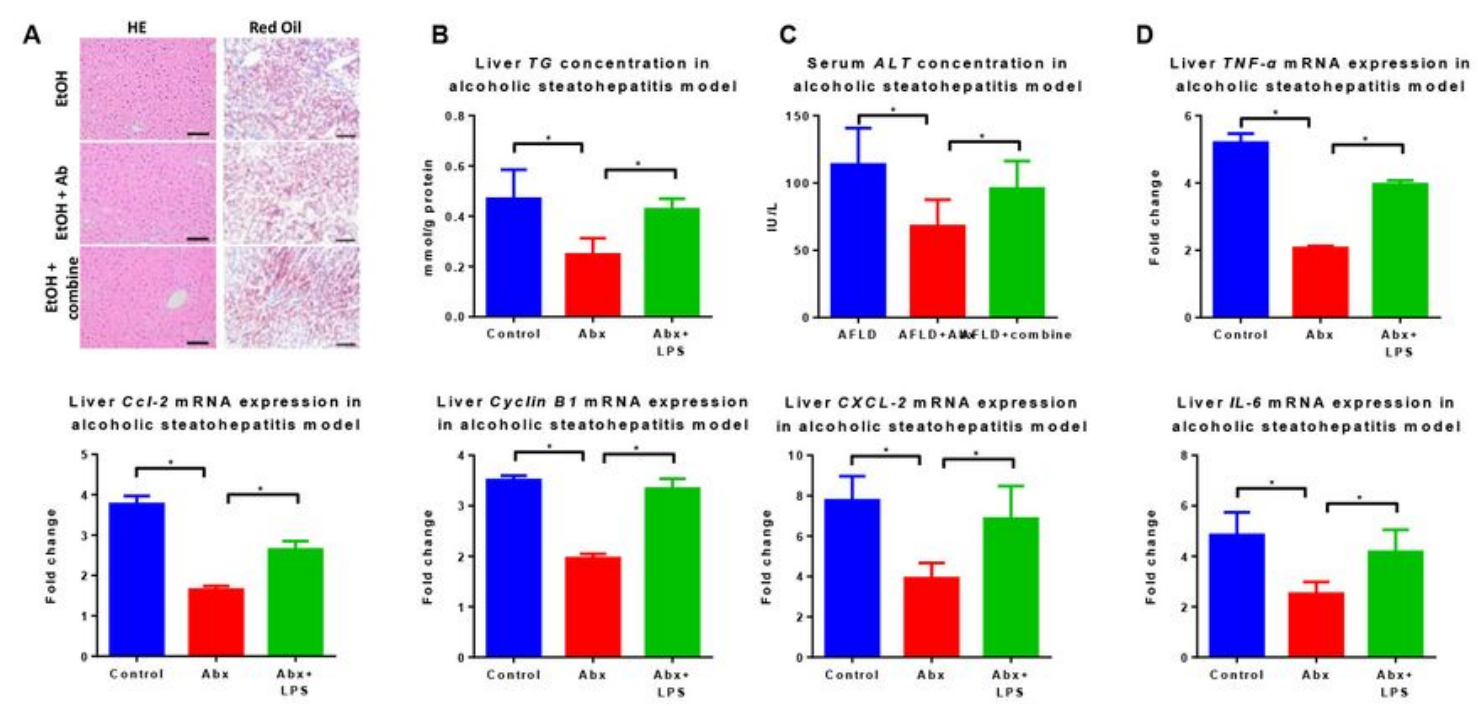

E
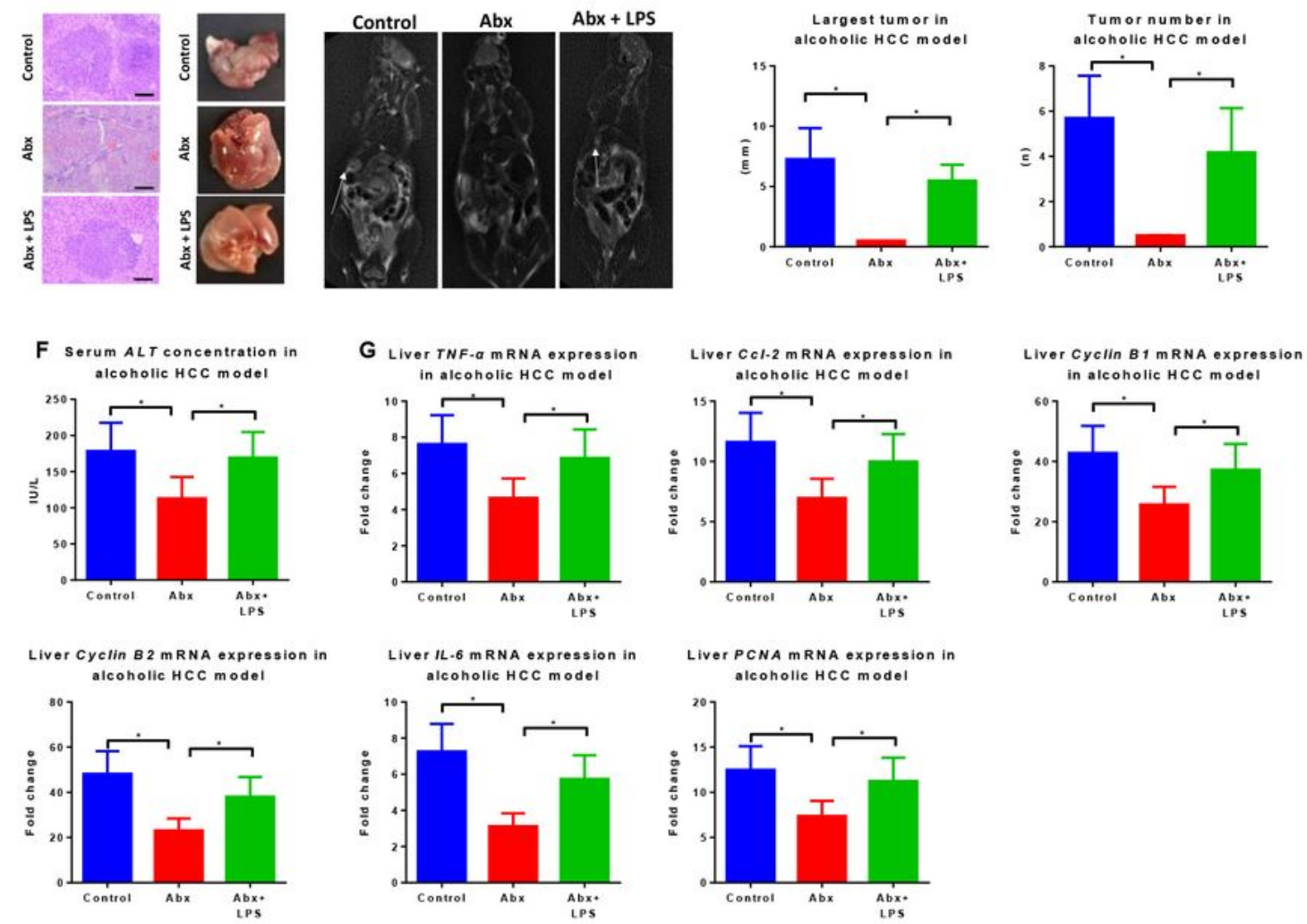

\section{Figure 4}

Intestinal LPS contributes to alcohol induced hepatosteatosis and subsequent HCC in mice. A: Hepatosteatosis was determined by HE and oil red staining after treatment with alcohol for 5 weeks. B-C: Liver TG and serum ALT concentrations were determined by ELISA in the mouse model of alcoholic hepatosteatosis. D: Liver levels of TNF-a, IL-6, cyclin B1, CXCL-2 and CCL-2 genes were determined by qPCR in the alcoholic hepatosteatosis model. E: Mouse liver tumors were presented by photography, HE 
staining and MRI scan after development of the alcoholic HCC model. F: The serum ALT concentration was determined by ELISA in alcoholic HCC mice. G: Liver levels of TNF-a, IL-6, cyclin B1, cyclin B2, CCL-2 and PCNA genes were determined by qPCR in the alcoholic HCC model.

Figure 5.
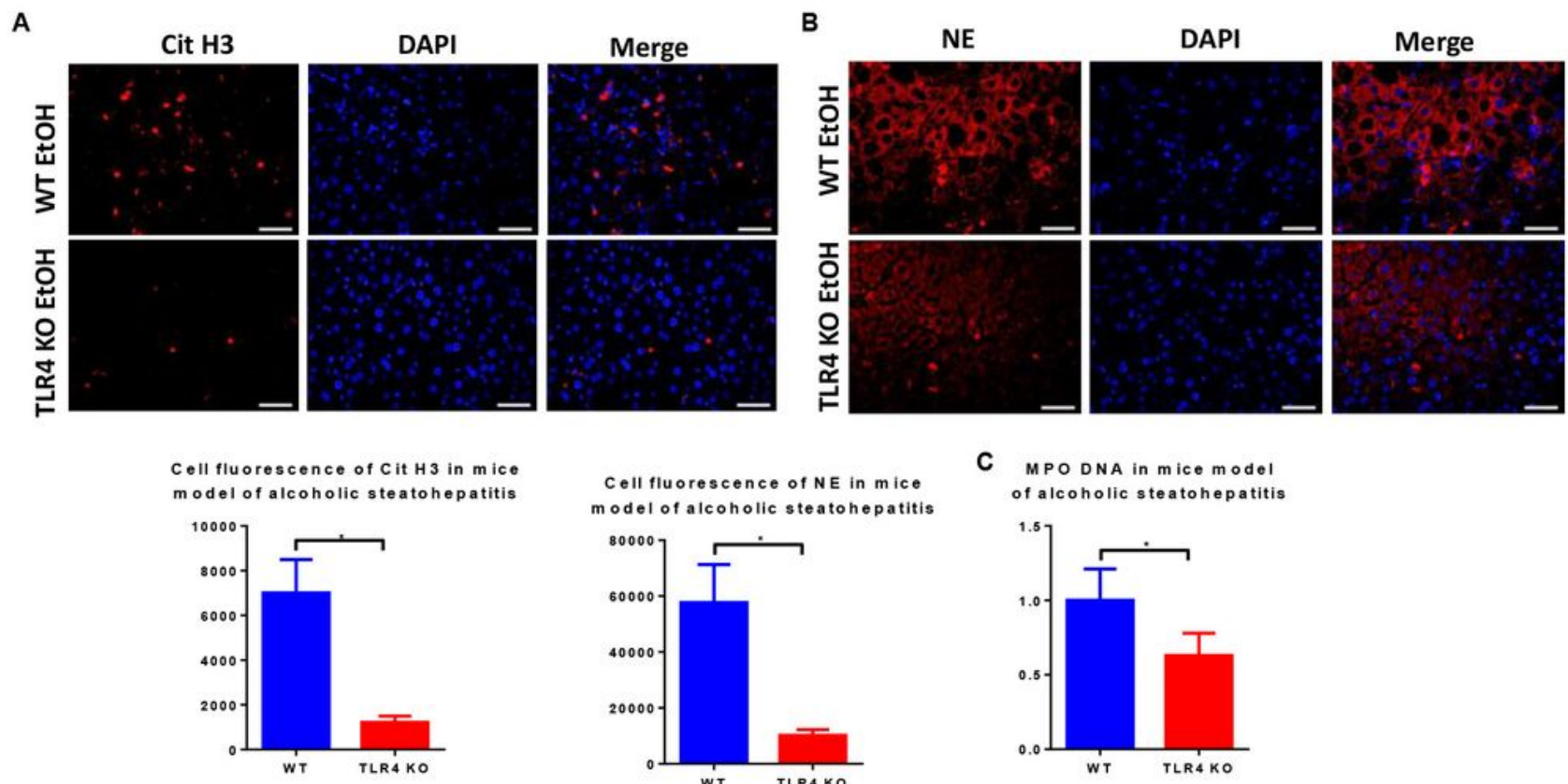

D

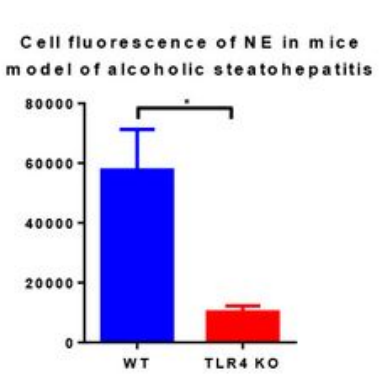

C MPODNA in mice model of alcoholic steatohepatitis
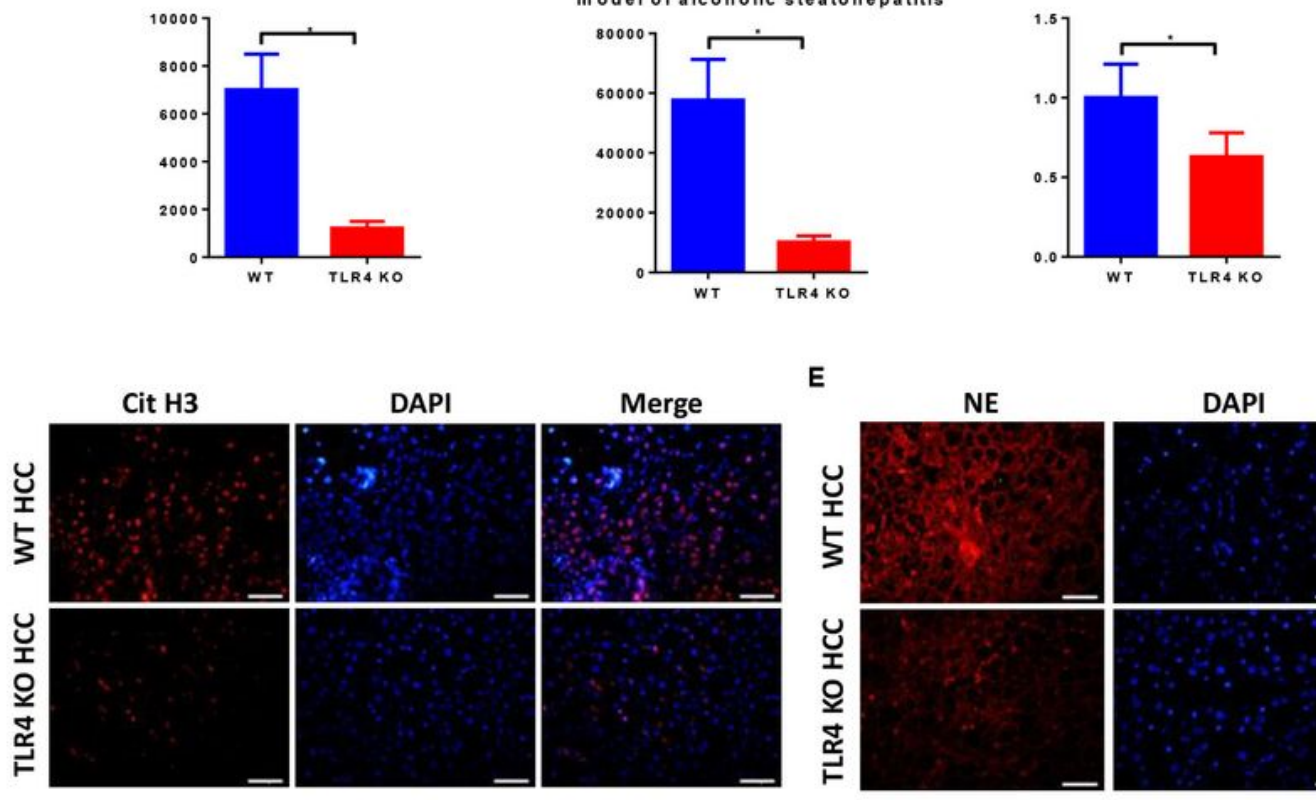

E

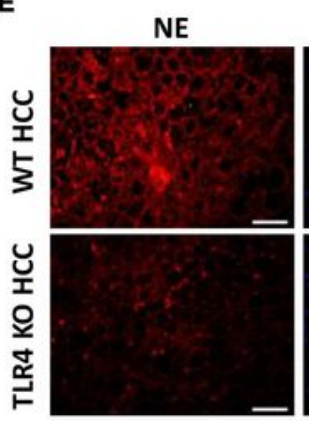

DAPI
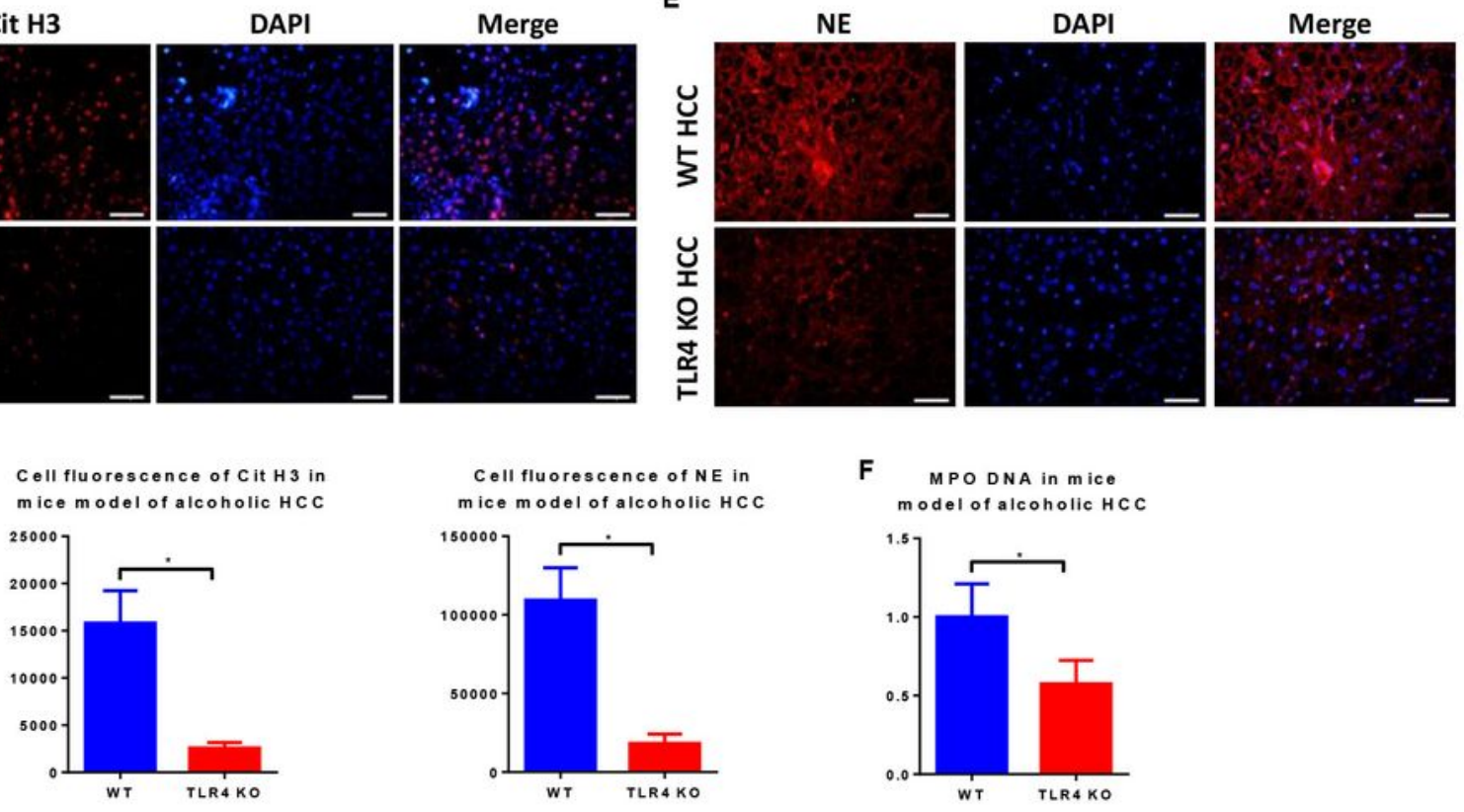

\section{Figure 5}

Lack of TLR4 results in less intrahepatic NET formation during the process of alcoholic hepatosteatosis and subsequent HCC in mice. A-B: Intrahepatic NETs were determined by immunofluorescence staining of 
cit H3 and NE in both WT and TLR4 KO mice after treatment with alcohol for 5 weeks. C: Liver MPO-DNA was tested by ELISA in both WT and TLR4 KO mice in a model of alcoholic hepatosteatosis. D-E: Livers from WT and TLR4 KO mice were tested for NETs by expression of cit $\mathrm{H} 3$ and NE, which were determined by immunofluorescence in the model of alcoholic HCC. F: Liver MPO-DNA was determined by ELISA in the alcoholic HCC model in both WT and TLR4 KO mice.

Figure 6.
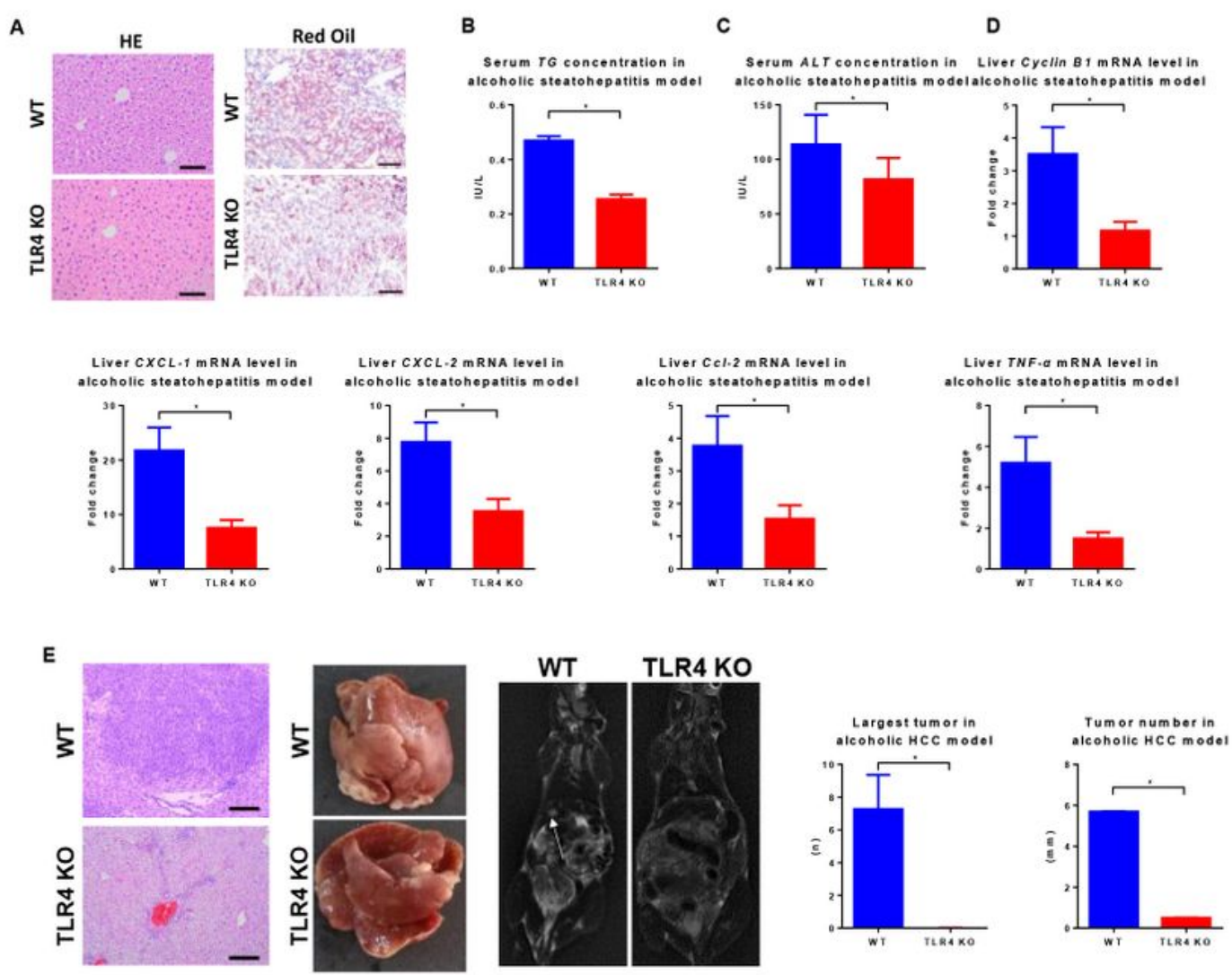

F
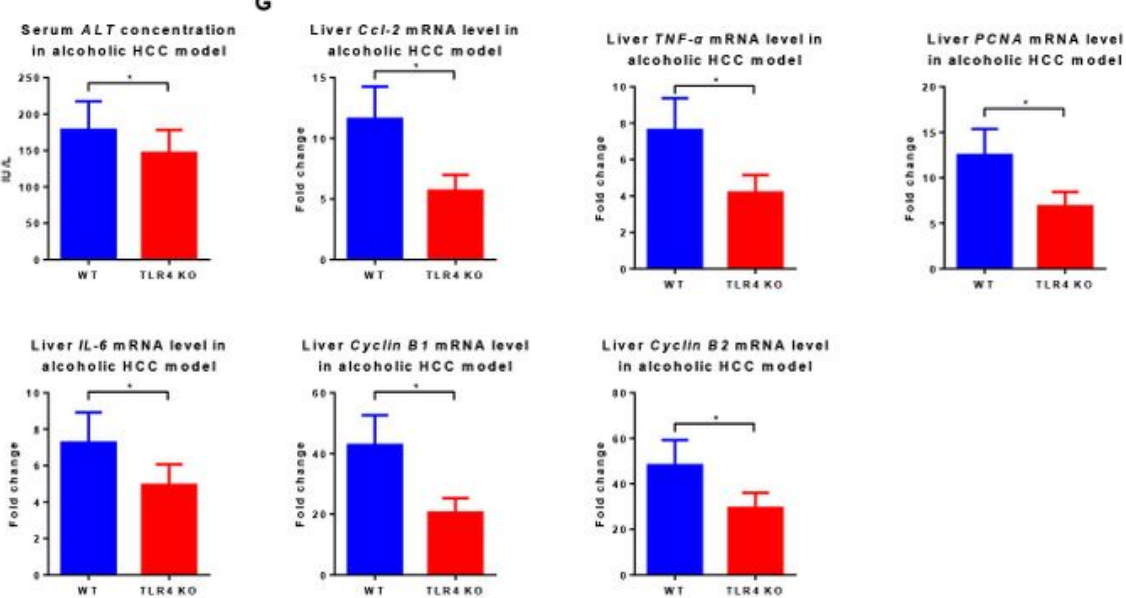

Figure 6 
Mice alcoholic hepatosteatosis and subsequent HCC are attenuated in the absence of TLR4. A: Hepatosteatosis was determined by HE and oil red staining after treatment with alcohol for 5 weeks in WT and TLR4 KO mice. B-C: Liver TG and serum ALT concentrations were determined by ELISA in WT and TLR4 KO mouse models of alcoholic hepatosteatosis. D: WT and TLR4 KO mouse liver levels of TNF-a, IL-6, cyclin B1, CXCL-2 and CCL-2 genes were determined by qPCR in the alcoholic hepatosteatosis model. E: WT and TLR4 KO mouse liver tumors are presented by photography, HE staining and MRI scanning after development of the alcoholic HCC model. F: Serum ALT concentrations were determined by ELISA in the alcoholic HCC model in WT and TLR4 KO mice. G: Liver levels of TNF-a, IL-6, cyclin B1, cyclin B2, CCL-2 and PCNA genes were determined by qPCR in alcoholic HCC model in WT and TLR4 KO backgrounds. 